\title{
Measuring Neutrino Oscillations with Nuclear Reactors
}

\author{
R. D. McKeown \\ W. K. Kellogg Radiation Laboratory, California Institute of Technology, Pasadena, CA
}

\begin{abstract}
Since the first direct observations of antineutrino events by Reines and Cowan in the 1950's [1], nuclear reactors have been an important tool in the study of neutrino properties. More recently, the study of neutrino oscillations has been a very active area of research. The pioneering observation of oscillations by the KamLAND experiment has provided crucial information on the neutrino mixing matrix. New experiments to study the remaining unknown mixing angle are currently under development. These recent studies and potential future developments will be discussed.
\end{abstract}

Keywords: Neutrino Mass; Neutrino Mixing; Neutrino Oscillations

PACS: $14.60 . \mathrm{Pq}, 26.65 .+\mathrm{t}, 28.50 . \mathrm{Hw}$

The standard model of particle physics contains (by hypothesis to reproduce the observed states) 3 generations of quarks and leptons. The quarks and charged leptons obtain their masses due to the Higgs mechanism, whereas the neutral leptons (neutrinos) are massless. The left-handed leptons belong to weak isospin doublets

$$
\psi_{L}=\left(\begin{array}{c}
v_{L} \\
\ell_{L}
\end{array}\right)
$$

whereas the corresponding right-handed charged lepton $\ell_{R}$ is a weak isosinglet

$$
\psi_{R}=\ell_{R} .
$$

The right-handed neutrinos do not appear in the standard model and there is no interaction that could generate such particles. The absence of the right-handed neutrino necessarily requires that $m_{v}=0$, since for $m_{v} \neq 0$ a left-handed neutrino could become right-handed by a Lorentz transformation.

It has been known for some time that, for the quarks, the mass eigenstates are not the same states as the flavor eigenstates produced in weak interactions. The flavor states are obtained by an SU(3) rotation of the mass eigenstates, described by a unitary rotation matrix known as the Cabibbo-Kobayashi-Maskawa (CKM) matrix. The CKM matrix is almost diagonal, and so it seems that there is some minor lack of alignment of the flavor and mass eigenstates. This slight rotation generates many interesting phenomena, including $\mathrm{CP}$ violation, but its origin is not understood at a fundamental level.

During the last decade, we have obtained evidence for much stronger mixing of the neutrino flavor states [2]. This requires that neutrinos have finite mass, and implies that the mechanism for generating misalignment of the mass and flavor eigenstates is of greater importance than for the quarks. In particular, the strong mixing and much lighter values of neutrino mass (relative to the charged fermions) supports the view that neutrino

CP947, VII Latin American Symposium on Nuclear Physics and Applications

edited by R. Alarcon, P. L. Cole, C. Djalali, and F. Umeres

(C) 2007 American Institute of Physics 978-0-7354-0461-8/07/\$23.00 
masses are generated by a very different mechanism than the Higgs mechanism. The "see-saw" mechanism [3] involving very heavy right-handed neutrinos is a commonly used example for generation of the anomalously light (relative to the charged fermions) neutrino masses. In any case, the existence of finite neutrino mass requires modification of the standard electroweak theory.

\section{NEUTRINO OSCILLATIONS}

The phenomenon of neutrino oscillations occurs when the flavor eigenstates (i.e., states produced in weak interaction processes, $v_{e}, v_{\mu}$ and $v_{\tau}$ ) are not identical with the mass eigenstates. The neutrino flavor eigenstate produced in a weak process (such as nuclear beta decay) is then a superposition of the mass eigenstates, and the subsequent evolution of the state as it propagates through space involves slippage of the relative phases of the mass eigenstates leading to flavor oscillations.

This is easily demonstrated in a 2 flavor approximation where there are 2 flavor eigenstates (e.g. $v_{e}$ and $v_{\mu}$ ) that are superpositions of 2 mass eigenstates ( $v_{1}$ and $v_{2}$, with masses $m_{1}$ and $m_{2}$ ). The mixing is described by a matrix involving one mixing angle $\theta$

$$
\left(\begin{array}{l}
v_{e} \\
v_{\mu}
\end{array}\right)=\left(\begin{array}{cc}
\cos \theta & \sin \theta \\
-\sin \theta & \cos \theta
\end{array}\right)\left(\begin{array}{l}
v_{1} \\
v_{2}
\end{array}\right) .
$$

If a $v_{e}$ is produced in an experiment, it will develop a component of $v_{\mu}$ as it propagates through space. The probability of observing a $v_{\mu}$ oscillates in the propagation distance $L$. It is straightforward to show that the probability for the $v_{\mu}$ state is given by

$$
P\left(v_{e} \rightarrow v_{\mu}\right)=\sin ^{2}(2 \theta) \sin ^{2}\left[1.27 \Delta m^{2}\left(\mathrm{eV}^{2}\right) \frac{L(\mathrm{~m})}{E_{v}(\mathrm{MeV})}\right],
$$

where $\Delta m^{2} \equiv\left|m_{2}^{2}-m_{1}^{2}\right|$ is the difference in squared masses and $E_{v}$ is the neutrino energy. Thus, these oscillations are characterized by the oscillation length

$$
L_{o s c}(\mathrm{~m})=\frac{2.48 E_{v}(\mathrm{MeV})}{\Delta m^{2}\left(\mathrm{eV}^{2}\right)}
$$

and by the amplitude $\sin ^{2} 2 \theta$.

The disappearance of the electron type neutrinos is given by the survival probability

$$
P\left(v_{e} \rightarrow v_{e}\right)=1-\sin ^{2}(2 \theta) \sin ^{2}\left[1.27 \Delta m^{2}\left(\mathrm{eV}^{2}\right) \frac{L(\mathrm{~m})}{E_{v}(\mathrm{MeV})}\right]
$$

This expression is appropriate for the description of the disappearance of electron antineutrinos from nuclear reactors, since the appearing muon neutrinos are below threshold for detection by charged current reactions. (Due to CPT invariance the behavior of neutrinos and antineutrinos will be the same.)

The 3 flavor case is a straightforward generalization in which there are 3 mass eigenstates (with masses $m_{1}, m_{2}$, and $m_{3}$ ) and a $3 \times 3$ mixing matrix that depends on 
3 mixing angles $\left(\theta_{12}, \theta_{23}\right.$, and $\left.\theta_{13}\right)$ plus a $C P$-violating phase $\delta_{C P}$. The mixing matrix is denoted $U_{P M N S}$ ( for Pontecorvo [4], Maki, Nakagawa and Sakata [5]) and, for neutrino oscillation physics, can be conveniently written as a product of three matrices:

$$
U_{P M N S}=\left(\begin{array}{ccc}
1 & 0 & 0 \\
0 & c_{23} & s_{23} \\
0 & -s_{23} & c_{23}
\end{array}\right) \times\left(\begin{array}{ccc}
c_{13} & 0 & s_{13} e^{-i \delta_{C P}} \\
0 & 1 & 0 \\
-s_{13} e^{i \delta_{C P}} & 0 & c_{13}
\end{array}\right) \times\left(\begin{array}{ccc}
c_{12} & s_{12} & 0 \\
-s_{12} & c_{12} & 0 \\
0 & 0 & 1
\end{array}\right)
$$

where $s_{i j} \equiv \sin \theta_{i j}$ and $c_{i j} \equiv \cos \theta_{i j}$. It is important to note that the term involving the $C P$-violating phase $s_{13} e^{-i \delta_{C P}}$ vanishes if $\theta_{13}=0$. Thus the angle $\theta_{13}$ can be viewed as the gateway to observation of $C P$ violation in the lepton sector.

The formula for disappearance of electron neutrinos (or antineutrinos) in the 3 flavor case becomes [6]

$$
\begin{aligned}
P\left(v_{e} \rightarrow v_{e}\right) & =1-\left\{\cos ^{4} \theta_{13} \sin ^{2} 2 \theta_{12}\left(1-\cos \Delta_{21}\right)\right. \\
& +\cos ^{2} \theta_{12} \sin ^{2} 2 \theta_{13}\left(1-\cos \Delta_{31}\right) \\
& \left.+\sin ^{2} \theta_{12} \sin ^{2} 2 \theta_{13}\left(1-\cos \Delta_{32}\right)\right\} / 2
\end{aligned}
$$

where $\Delta_{i j} \equiv \Delta m_{i j}^{2} L / 2 E_{v}$. This is the general 3-flavor expression relevant to antineutrino disappearance from nuclear reactors. Note that the 3 terms inside the brackets oscillate with different "frequencies" depending on the values of the $\Delta m_{i j}$. Thus one can choose the baseline $L$ to maximize (or minimize) the sensitivity to particular $\Delta m_{i j}$.

\section{REACTOR ANTINEUTRINO EXPERIMENTS}

The pioneering work of Reines and Cowan in the 1950's established the basic methodology for measuring antineutrinos from nuclear reactors, and actually led to the first observation of antineutrinos. The antineutrinos are produced by beta decays of neutron rich fission products in the reactor core. The primary contributions are from ${ }^{235} \mathrm{U}$ and ${ }^{239} \mathrm{Pu}$, for which there are laboratory measurements of the $\beta$ spectra from the fission products. A minor $(<10 \%)$ contribution from ${ }^{238} \mathrm{U}$ is obtained from calculation. Generally, one obtains $6 \bar{v}_{e}$ per fission, which can be combined with the $200 \mathrm{MeV}$ per fission energy release to yield $\sim 2 \times 10^{20} \bar{v}_{\mathrm{e}} \mathrm{s}^{-1} \mathrm{GW}_{\mathrm{th}}^{-1}$. So the total antineutrino flux is well-constrained by the total thermal power of a reactor, and is, of course, isotropically distributed. The energy spectrum of the $\bar{v}_{e}$ is a steeply falling spectrum (see Fig. 1) and depends slightly on the mix of fissionable material which varies during the fuel cycle of the reactor. Generally, the flux and spectrum can be predicted to the $\sim 2 \%$ accuracy.

Detection of the antineutrinos is generally accomplished via the inverse beta decay reaction on the proton:

$$
\bar{v}_{e}+p \rightarrow e^{+}+n \text {. }
$$

The threshold for this reaction is $E_{V} \simeq 1.8 \mathrm{MeV}$, and the cross section increases roughly quadratically with $E_{v}$ above this value, as shown in Fig. 1 . The inverse beta decay cross section is well-constrained by the measured neutron lifetime, and the estimated uncertainty is below $0.5 \%$ [7]. The interactions in the detector are proportional to the 
product of cross section and flux, which produces a broad spectrum peaked at about $4 \mathrm{MeV}$ in antineutrino energy.

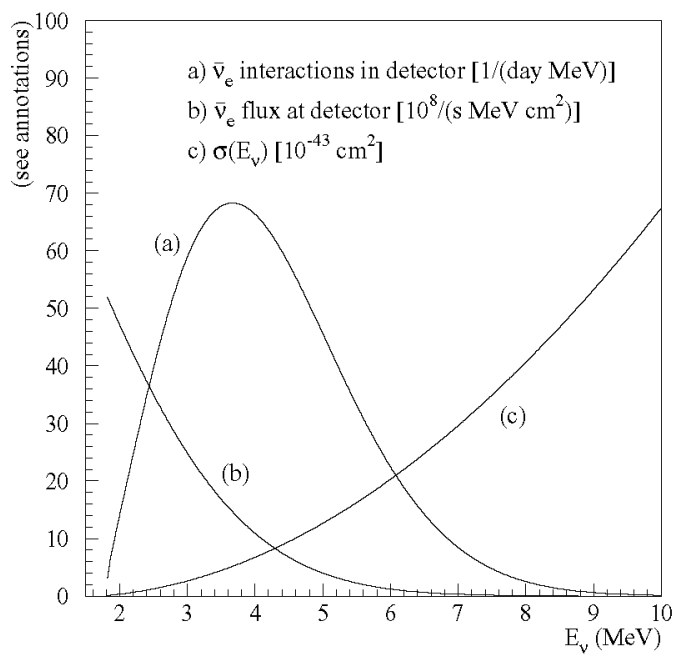

FIGURE 1. Reactor $\bar{v}_{e}$ spectrum, inverse beta decay cross section, and $\bar{v}_{e}$ interaction spectrum, from [8].

For the inverse beta decay reaction Eq. 9, one can generally detect the positron (kinetic energy plus annihilation into two $0.511 \mathrm{MeV}$ gamma rays) followed by the capture of the thermalized neutron. The total positron energy $E_{e^{+}}$is closely related to the incident antineutrino energy $E_{v}$

$$
E_{v} \simeq E_{e^{+}}+1.3 \mathrm{MeV}+E_{n}
$$

where $E_{n}$ is the neutron recoil kinetic energy which is quite small ( $E_{n} \sim 0.03 \mathrm{MeV}$ ). The antineutrino flux and energy spectrum have been measured using this method in many experiments close to reactor cores (i.e., $L \ll E_{v} / \Delta m_{i j}^{2}$ where oscillations are not yet present), and one generally finds excellent agreement at the $\sim 2 \%$ level.

\section{CHOOZ and Palo Verde}

In 1998, the Super-Kamiokande collaboration reported the observation of neutrino oscillations in atmospheric neutrinos (produced by cosmic rays in the upper atmosphere). The atmospheric neutrino data indicated that $\Delta m^{2} \sim 3 \times 10^{-3} \mathrm{eV}^{2}$ with a large mixing angle $\sin ^{2} 2 \theta \sim 1$, and that the observed $v_{\mu}$ disappearance was not predominantly $v_{\mu} \rightarrow v_{e}$. Two reactor neutrino experiments were built to further study this phenomenon and search for $\bar{v}_{e}$ disappearance. One was at the reactor plant in Chooz, France [9] with a baseline of $L \simeq 1 \mathrm{~km}$ and the other was at the Palo Verde plant in Arizona, USA [10] with a baseline of $L \simeq 0.8 \mathrm{~km}$. Both experiments detected the predicted flux of antineu- 
trinos without oscillations, implying an upper limit on the value of $\sin ^{2} 2 \theta<0.13$ for $\Delta m^{2}=2.8 \times 10^{-3} \mathrm{eV}^{2}$ at $90 \%$ confidence.

\section{KamLAND}

The Kamioka Liquid scintillator AntiNeutrino Detector, KamLAND, was the first reactor neutrino experiment to observe neutrino oscillations. Numerous previous solar neutrino observations indicated a deficit of neutrinos from the sun, relative to the standard solar model predictions [11]. If interpreted as matter-enhanced flavor oscillations ("MSW effect"), this deficit implied a much smaller $\Delta m^{2} \sim 10^{-4} \mathrm{eV}^{2}$. Such a small $\Delta m^{2}$ implied a distance for maximum oscillation probability of $L \sim 100 \mathrm{~km}$. The loss of $\sim 30,000$ in flux due to such a long baseline (relative to CHOOZ and Palo Verde) was mitigated in KamLAND by the larger detector mass (1 kton) and by utilizing many (more than 50) power reactors situated in Japan. The baseline distance distribution of expected flux from these reactors displays a strong peak near $180 \mathrm{~km}$, as shown in Fig. 2 .

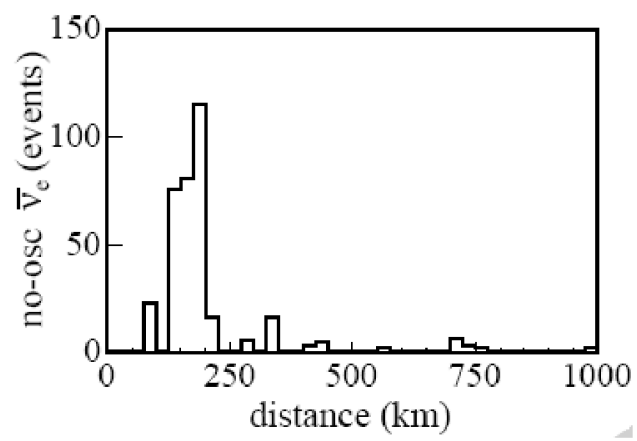

FIGURE 2. Predicted distribution of events in KamLAND vs. baseline distance from Japanese power reactors, assuming no neutrino oscillations, from [12].

The KamLAND collaboration has reported [12] a significant $(>4 \sigma)$ deficit in the observed flux of antineutrinos relative to the no-oscillation hypothesis

$$
\frac{N_{o b s}}{N_{n o-o s c}}=0.658 \pm 0.044 \pm 0.047 \text {. }
$$

In addition, KamLAND obtained a measurement of the $\bar{v}_{e}$ energy spectrum showing a substantial distortion compared to the no-oscillation spectrum. When plotted as a function of the ratio $L_{0} / E_{V}$, with the reference baseline distance $L_{0} \equiv 180 \mathrm{~km}$, the spectrum shows an impressive oscillation pattern(see Fig. 3). The best fit to the KamLAND data yields a value of $\Delta m^{2}=7.9_{-0.5}^{+0.6} \times 10^{-5} \mathrm{eV}^{2}$. Assuming CPT invariance, one can combine this information with the solar neutrino data (particularly the data from the Sudbury Neutrino Observatory, or SNO) [13] to obtain

$$
\begin{aligned}
\Delta m_{12}^{2} & =7.9_{-0.5}^{+0.6} \times 10^{-5} \mathrm{eV}^{2} \\
\tan ^{2} \theta_{12} & =0.40_{-0.07}^{+0.10} .
\end{aligned}
$$


Note that, since we now have oscillations involving all three flavors of neutrinos, we have introduced the notation $\Delta m_{12}^{2}$ and $\theta_{12}$ to designate this oscillation mode.

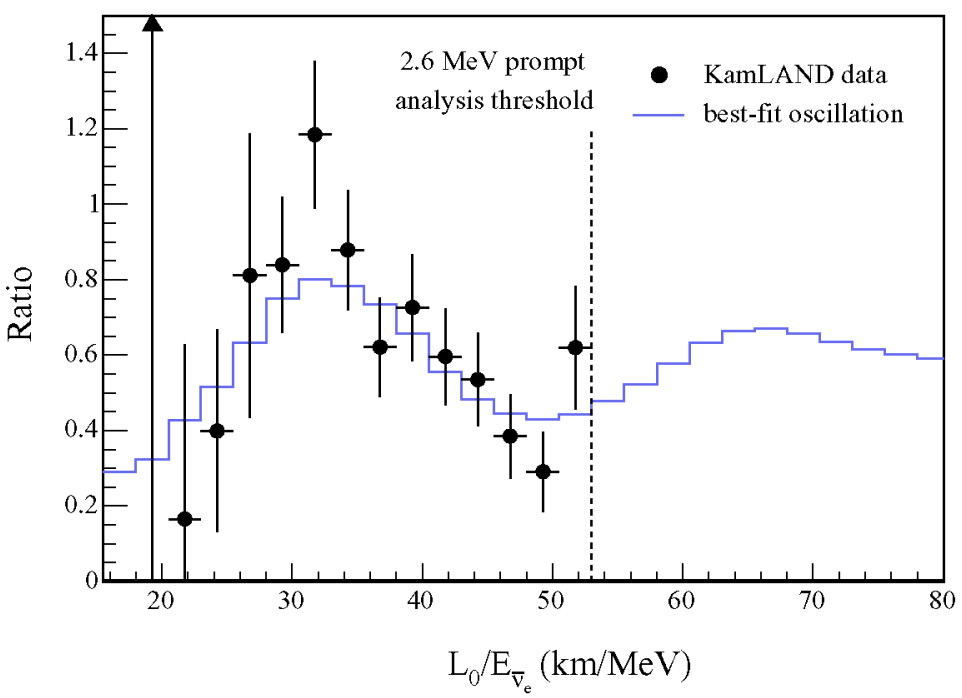

FIGURE 3. Distribution of observed events in KamLAND vs. $L_{0} / E_{v}[12]$, where $L_{0} \equiv 180 \mathrm{~km}$. The histogram is the best fit to the neutrino oscillation hypothesis.

\section{FUTURE EXPERIMENTS}

The present situation regarding the neutrino oscillation parameters can be summarized as follows. We now have good knowledge of $\Delta m_{12}^{2}$ and $\theta_{12}$ (Eq. 13) from KamLAND and the solar neutrino experiments. The Super-Kamiokande atmospheric neutrino data [14] yield $\left(1.9<\Delta m_{32}^{2}<3.0\right) \times 10^{-3} \mathrm{eV}^{2}$ and $\sin ^{2}\left(2 \theta_{23}\right)>0.92$ at $90 \% \mathrm{CL}$. The MINOS long baseline accelerator neutrino experiment [15] recently reported $\Delta m_{32}^{2}=$ $2.74_{-0.26}^{+0.44} \times 10^{-3} \mathrm{eV}^{2}$ and $\sin ^{2}\left(2 \theta_{23}\right)>0.87$ at $68 \% \mathrm{CL}$. Since $\Delta m_{12}^{2} \ll \Delta m_{32}^{2}$, we also know $\Delta m_{31}^{2} \simeq \Delta m_{32}^{2}$. So we now have good knowledge of all the $\Delta m_{i j}^{2}$ and two of the mixing angles $\theta_{12}$ and $\theta_{23}$. However, we only have the upper limit for $\theta_{1} 3$ from CHOOZ and Palo Verde. As a result, there are new experiments being planned to determine this last mixing angle in the neutrino mixing matrix. As mentioned above, this parameter is also key for future attempts to study the $C P$-violating phase $\delta_{C P}$.

There are presently four new $\theta_{13}$ reactor antineutrino experiments being pursued. These all intend to utilize the method of comparing two identical detectors at different baselines to reduce systematic errors, as first proposed by Mikaelyan and Sinev [16]. The most advanced are a new experiment at the CHOOZ site known as Double-CHOOZ [17], and an experiment at a new site in China, the Daya Bay experiment [18]. The other 
two experiments are RENO in Korea [19], and Angra in Brazil [20]. The Daya Bay experiment has the best projected sensitivity for $\theta_{13}$.

\section{The Daya Bay Experiment}

The Daya Bay nuclear power plant currently operates 4 reactors with a total thermal power of $11.6 \mathrm{GW}_{\mathrm{th}}$. Two additional reactors will be constructed during the next three years, increasing the total power to $17.4 \mathrm{GW}_{\mathrm{th}}$. The reactors are located in 2 groups with a pair at the "Daya Bay" location and the remaining at the "Ling Ao" location at about $1 \mathrm{~km}$ distance. The plant is located adjacent to mountainous terrain that facilitates construction of underground detector halls to reduce background from cosmic radiation. The experimental layout is shown in Fig. 4.

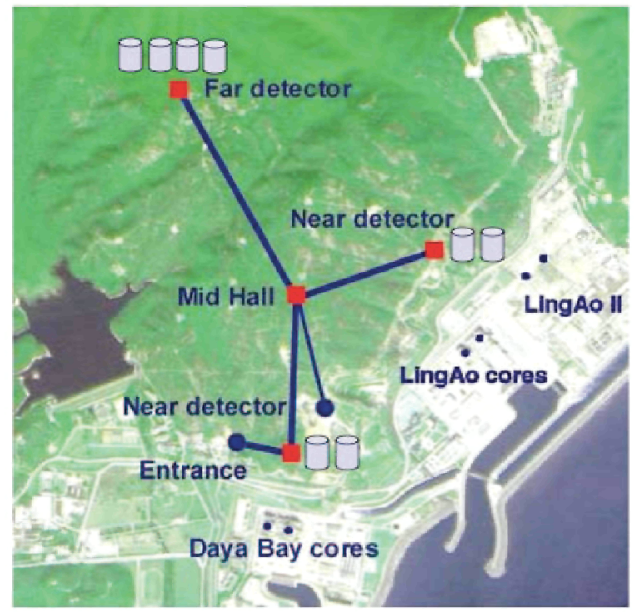

FIGURE 4. Default configuration of the Daya Bay experiment, optimized for best sensitivity in $\sin ^{2} 2 \theta_{13}$. Four detector modules are deployed at the far site and two each at each of the near sites.

The Daya Bay experiment will consist of eight detector modules, each with a target mass of 20 Tonnes. These detectors will be deployed in three experimental halls, connected by horizontal tunnels, as shown in Fig. 4. The cylindrical detector target consists of Gd-loaded liquid scintillator, surrounded by a liquid scintillator gamma catcher (to improve the efficiency for detection of the neutron capture on Gd), surrounded by a mineral oil buffer (for background reduction). 192 8-inch photomultipliers view each detector, and reflective sheets increase the light collection to obtain energy resolution of $\sim 15 \% / \sqrt{E(\mathrm{MeV})}$.

The projected sensitivity of the Daya Bay experiment is $\sin ^{2} 2 \theta_{13}<0.008$ at $90 \%$ confidence for three years of running. The excavation of the underground tunnels and experimental halls will begin in Fall 2007 and will require about 2 years. The full experiment should be operational in late 2010 . 


\section{ACKNOWLEDGMENTS}

I would like to thank all my collaborators on the KamLAND experiment and the Daya Bay project for many fruitful discussions. I am particularly indebted to Petr Vogel for much enlightenment and his comments on this manuscript.

\section{REFERENCES}

1. C. L. Cowan, Jr., F. Reines, F. B. Harrison, H. W. Kruse, and A. D. McGuire, Science 124, 103 (1956); F. Reines and C. L. Cowan, Phys. Rev. 92, 830 (1953).

2. R. D. McKeown and P. Vogel, Phys. Rep. 394315 (2004).

3. M. Gell-Mann, P. Ramond and R. Slansky, in Supergravity, D. Freedman and P. van Nieuwenhuizen eds., North Holland, Amsterdam 1979; T. Yanagida, in Proc. of the Workshop on Unified Theory amd Baryon Number of the Universe, O. Sawada and A. Sugamoto eds., KEK, Japan (1979); R. N. Mohapatra and G. Senjanovic, Phys. Rev. Lett. 44, 912 (1980).

4. B. Pontecorvo, Sov. Phys. JETP 6, 429 (1958); B. Pontecorvo, Sov. Phys. JETP 33, 549 (1967).

5. Z. Maki, M. Nakagawa and S. Sakata, Prog. Theor. Phys. 28, 870 (1962).

6. V. Barger, D. Marfatia and K. Whisnant, Int. J. Mod. Phys. E12, 569-647 (2003).

7. P. Vogel and J. F. Beacom, Phys. Rev. D60, 053003 (1999); A. Kurylov, M.J. Ramsey-Musolf, and P. Vogel, Phys.Rev. C67 035502 (2003).

8. C. Bemporad, G. Gratta and P. Vogel, Rev. Mod. Phys. 74, 297 (2002).

9. M. Appolonio et al., Eur. Phys. J. C27, 331 (2003)

10. F. Boehm, et al., Phys. Rev. D64, 112001 (2001).

11. J.N. Bahcall and R. Davis, Science 191, 264 (1976), J.N. Bahcall, Neutrino Astrophysics, Cambridge University Press, 1989, J.N. Bahcall, Astrophys. J. 467, 475 (1996).

12. T. Araki, et al., Phys. Rev. Lett. 94, 081801 (2005).

13. S. N. Ahmaed, et al., Phys. Rev. Lett. 92, 181301 (2004).

14. Y. Ashie et al., Phys. Rev. D71, 112005 (2005).

15. D. G. Michael, et al., Phys. Rev. Lett. 97, 191801 (2006).

16. L. A. Mikaelyan and V. V. Sinev, Phys. Atomic Nucl. 63, 1002 (2000).

17. F. Ardellier et al., Double Chooz: A Search for the Neutrino Mixing Angle $\theta_{13}$, arXiv:hep-ex/0606025 (2006).

18. Daya Bay Collaboration, A Precision Measurement of the Neutrino Mixing Angle $\boldsymbol{\theta}_{13}$ using Reactor Antineutrinos at Daya Bay, hep-ex/0701029 (2007).

19. K.K.Joo for the RENO collaboration, Nucl. Phys. B168, 125 (2007).

20. http://www.hep.anl.gov/minos/reactor13/Angra/proposal.html 